\title{
BIFURCATIONS OF AFFINE INVARIANTS FOR ONE-PARAMETER FAMILY OF GENERIC CONVEX PLANE CURVES
}

\author{
TAKASHI SANO \\ Department of Mathematics, Faculty of Science, Hokkaido University \\ Sapporo 060-0810, Japan \\ E-mail: t-sano@math.sci.hokudai.ac.jp
}

\begin{abstract}
We study affine invariants of plane curves from the view point of the singularity theory of smooth functions. We describe how affine vertices and affine inflexions are created and destroyed.
\end{abstract}

1. Introduction. In [8] we have studied affine invariants for generic convex plane curves as an application of the singularity theory for affine invariant functions. We have introduced the notion of affine distance cubed-functions and affine height functions on convex plane curves and characterized affine vertices and affine inflexions by using these functions. Affine vertices and affine inflexions have been classically known as sextactic points and parabolic points, respectively. It has been known that the sextactic point and the parabolic point are equi-affine invariants. We now have the following natural question: How are affine vertices and affine inflexions created and destroyed? In this note, we attempt to give an answer to the question. The basic tools we use here are families of affine distance-cubed functions and affine height functions.

This paper is divided into four sections. The main result is Theorem 2 which is formulated in Section 2. The proof of Theorem 2 is given in Section 3. We shall give some examples with pictures which illustrate the results of Theorem 2 in Section 4. The basic techniques we use in this paper is based on the paper of J. W. Bruce [3].

All curves and maps considered here are of class $C^{\infty}$ unless otherwise stated.

2. Basic notions. For the basic notions and classical results in affine differential geometry, see $[2,8,9]$.

Let $\mathbb{R}^{\not}$ be an affine plane which adopts coordinates such that the area of the parallelogram spanned by two vectors $a=\left(a_{1}, a_{2}\right), b=\left(b_{1}, b_{2}\right)$ is given by the determinant of

1991 Mathematics Subject Classification: Primary 53A15; Secondary 58C27.

The paper is in final form and no version of it will be published elsewhere. 
$a$ and $b$, that is $|a \quad b|=a_{1} b_{2}-a_{2} b_{1}$. Let $S^{1}$ be the unit circle in $\mathbb{R}^{\not \models}$, and $\gamma: S^{1} \longrightarrow \mathbb{R}^{\not \models}$ be a smooth plane curve with $|\dot{\gamma}(t) \ddot{\gamma}(t)|>0$, where $\dot{\gamma}(t)=\frac{d \gamma}{d t}(t)$. If we reparametrize a given curve $\gamma$ by using $s(t)=\int_{t_{0}}^{t}|\dot{\gamma}(t) \ddot{\gamma}(t)|^{\frac{1}{3}} d t$, then the curve satisfies the condition $\left|\gamma^{\prime}(s) \gamma^{\prime \prime}(s)\right|=1$, where $\gamma^{\prime}(s)=\frac{d \gamma}{d s}(s)$. We call such a parameter $s$ an affine (arc-length) parameter. We call $\gamma^{\prime}(s)$ an affine tangent vector and $\gamma^{\prime \prime}(s)$ an affine normal vector. The affine curvature is defined by $\kappa(s)=\left|\gamma^{\prime \prime}(s) \gamma^{\prime \prime \prime}(s)\right|$.

We define the notion of affine vertices as follows. We say that the point $\gamma\left(s_{0}\right)$ of curve $\gamma$ is an affine vertex of order $k-1$ if $\kappa^{\prime}\left(s_{0}\right)=\kappa^{\prime \prime}\left(s_{0}\right)=\ldots=\kappa^{(k-1)}\left(s_{0}\right)=0$ and $\kappa^{(k)}\left(s_{0}\right) \neq 0$. We also say that the order of the affine vertex is $k-1$. In particular, the affine vertex of order 1 is called the ordinary affine vertex. If $\gamma\left(s_{0}\right)$ is not an affine vertex, we call the point $\gamma\left(s_{0}\right)$ the affine vertex of order 0 , or the order of the affine vertex is 0 .

We define the notion of affine inflexions as follows. We say that the point $\gamma\left(s_{0}\right)$ of curve $\gamma$ is an affine inflexion of order $k$ if $\kappa\left(s_{0}\right)=\kappa^{\prime}\left(s_{0}\right)=\ldots=\kappa^{(k-1)}\left(s_{0}\right)=0$ and $\kappa^{(k)}\left(s_{0}\right) \neq 0$. We also say that the order of the affine inflexion is $k$. In particular, the affine inflexion of order 1 is called the ordinary affine inflexion. If $\gamma\left(s_{0}\right)$ is not affine inflexion, we call the point $\gamma\left(s_{0}\right)$ the affine inflexion of order 0 , or the order of the affine inflexion is 0 .

We assume that $\gamma$ has the following properties, which are satisfied generically (cf. [5]).

(A-1) There is no conic having greater than six-point contact with $\gamma\left(S^{1}\right)$.

(A-2) The number of points $p$ of $\gamma\left(S^{1}\right)$ where the unique non-singular conic touching $\gamma\left(S^{1}\right)$ at $p$ with at least five-point contact is a parabola is finite.

(A-3) There is no parabola having six-point contact with $\gamma\left(S^{1}\right)$.

In [8], we have shown the following theorem.

THEOREM $1([8])$. Let $\gamma: S^{1} \longrightarrow \mathbb{R}^{\not \models}$ be a smooth plane curve with $\left|\gamma^{\prime}(s) \quad \gamma^{\prime \prime}(s)\right|>0$ satisfying (A-1)-(A-3). Then:

(1) Let $p$ be a point of the affine evolute of $\gamma$ at $s_{0}$, then locally at $p$, the affine evolute is

(a) diffeomorphic to the line in $\mathbb{R}^{\not}$ if the point $\gamma\left(s_{0}\right)$ is not the affine vertex of $\gamma$;

(b) diffeomorphic to the ordinary cusp in $\mathbb{R}^{\not}$ if the point $\gamma\left(s_{0}\right)$ is the ordinary affine vertex of $\gamma$.

(2) Let $p$ be a point of the affine normal curve of $\gamma$ at $s_{0}$. Then, locally at $p$, the affine normal curve is

(a) diffeomorphic to the line in $\mathbb{R}^{\not}$ if the point $\gamma\left(s_{0}\right)$ is not the affine inflexion of $\gamma$;

(b) diffeomorphic to the ordinary cusp in $\mathbb{R}^{\not \neq}$ if the point $\gamma\left(s_{0}\right)$ is the ordinary affine inflexion of $\gamma$.

The ordinary cusp is a curve which is given by $C=\left\{\left(x_{1}, x_{2}\right) \in \mathbb{R}^{\not} \mid x_{1}^{2}=x_{2}^{3}\right\}$.

Theorem 1 means that the affine vertex of a convex curve is characterized by the singularity of the affine evolute of convex curve and the affine inflexion of a convex curve is characterized by the singularity of the affine normal curve of convex curve. 
Here, we introduce some notation to describe the main theorem.

Let $U$ be an open interval $(-1,2)$. We consider the set

$$
\operatorname{Imm}^{+}\left(S^{1}, \mathbb{R}^{\not \models}\right)=\left\{i: S^{1} \longrightarrow \mathbb{R}^{\not \models} \mid i \text { is an immersion, }\left|\frac{d i}{d s}(s) \frac{d^{2} i}{d s^{2}}(s)\right|>0\right\} .
$$

We also consider the set

$$
\mathcal{C}=\left\{\Gamma: S^{1} \times U \longrightarrow \mathbb{R}^{\not \models} \mid \Gamma_{u} \in \operatorname{Imm}^{+}\left(S^{1}, \mathbb{R}^{\not \nvdash}\right) \text { for any } u \in U\right\} .
$$

In particular, $\Gamma_{0}$ and $\Gamma_{1}$ satisfy the above conditions (A-1), (A-2) and (A-3), and $\Gamma_{u}(s)$ satisfy $\left|\frac{d \Gamma_{u}}{d s}(s) \frac{d^{2} \Gamma_{u}}{d s^{2}}(s)\right|=1$ for all $u \in U$.

For any $\Gamma \in \mathcal{C}$, we define $\mathcal{A N}_{\Gamma}$ and $\mathcal{A} \mathcal{E}_{\Gamma}$ as follows:

$$
\begin{aligned}
& \mathcal{A} \mathcal{N}_{\Gamma}=\left\{\left(u, \frac{\partial^{2} \Gamma}{\partial s^{2}}(s, u)\right) \in U \times \mathbb{R}^{\not \nvdash} \mid s \in S^{1}\right\} \\
& \mathcal{A} \mathcal{E}_{\Gamma}=\left\{\left(u, \Gamma(s, u)+\frac{1}{\kappa(s, u)} \frac{\partial^{2} \Gamma}{\partial s^{2}}(s, u)\right) \in U \times \mathbb{R}^{\not \models} \mid \kappa(s, u) \neq 0, s \in S^{1}\right\} .
\end{aligned}
$$

Let $F_{i}: \mathbb{R} \times \mathbb{R}^{\backslash}, 0 \longrightarrow \mathbb{R}, 0$ be smooth function germs and $\left(X_{i}, 0\right)$ be set germs in $\left(\mathbb{R} \times \mathbb{R}^{\backslash}, 0\right)$, where $i=1,2$. We say that $\left(F_{1}, X_{1}\right)$ and $\left(F_{2}, X_{2}\right)$ are $\mathcal{R}$-equivalent if there exists a diffeomorphism germ $\Phi: \mathbb{R} \times \mathbb{R}^{\backslash}, 0 \longrightarrow \mathbb{R} \times \mathbb{R}^{\backslash}, 0$ such that $\Phi\left(X_{1}\right)=X_{2}$ and $\left.F_{1}\right|_{X_{1}}=\left.F_{2} \circ \Phi\right|_{X_{1}}$.

Let $\pi_{U}: U \times \mathbb{R}^{\not t} \longrightarrow U$ be the canonical projection. The following result is the main result in this paper.

THEOREM 2. There exists a dense subset $\mathcal{O} \subset \mathcal{C}$ such that for any $\Gamma \in \mathcal{O}$, we have

(1) Suppose that $\Gamma_{u_{0}}\left(s_{0}\right)$ is the affine inflexion of order $k$, then $k \leq 2$.

(a) If $k=0$, then the pair of germs $\left(\pi_{U}, \mathcal{A N}_{\Gamma}\right)$ at $\left(u_{0}, n_{\Gamma_{u_{0}}}\left(s_{0}\right)\right)$ is $\mathcal{R}$-equivalent to the pair of germs $\left(\pi_{1}, \mathbf{P}\right)$ at $(0,0)$;

(b) If $k=1$, then the pair of germs $\left(\pi_{U}, \mathcal{A N}_{\Gamma}\right)$ at $\left(u_{0}, n_{\Gamma_{u_{0}}}\left(s_{0}\right)\right)$ is $\mathcal{R}$-equivalent to the pair of germs $\left(\pi_{1}, \mathbf{C}\right)$ at $(0,0)$;

(c) If $k=2$, then the pair of germs $\left(\pi_{U}, \mathcal{A N}_{\Gamma}\right)$ at $\left(u_{0}, n_{\Gamma_{u_{0}}}\left(s_{0}\right)\right)$ is $\mathcal{R}$-equivalent to the pair of germs $\left(\pi_{1}, \mathbf{T}\right)$ at $(0,0)$.

(2) Suppose that $\Gamma_{u_{0}}\left(s_{0}\right)$ is the affine vertex of order $k$, then $k \leq 2$.

(a) If $k=0$, then the pair of germs $\left(\pi_{U}, \mathcal{A E}_{\Gamma}\right)$ at $\left(u_{0}, e_{\Gamma_{u_{0}}}\left(s_{0}\right)\right)$ is $\mathcal{R}$-equivalent to the pair of germs $\left(\pi_{1}, \mathbf{P}\right)$ at $(0,0)$;

(b) If $k=1$, then the pair of germs $\left(\pi_{U}, \mathcal{A E}_{\Gamma}\right)$ at $\left(u_{0}, e_{\Gamma_{u_{0}}}\left(s_{0}\right)\right)$ is $\mathcal{R}$-equivalent to the pair of germs $\left(\pi_{1}, \mathbf{C}\right)$ at $(0,0)$;

(c) If $k=2$, then the pair of germs $\left(\pi_{U}, \mathcal{A} \mathcal{E}_{\Gamma}\right)$ at $\left(u_{0}, e_{\Gamma_{u_{0}}}\left(s_{0}\right)\right)$ is $\mathcal{R}$-equivalent to the pair of germs $\left(\pi_{1}, \mathbf{T}\right)$ at $(0,0)$,

where $\mathbf{P}=\left\{\left(t, x_{1}, x_{2}\right) \mid x_{2}=0\right\}, \mathbf{C}=\left\{\left(t, x_{1}, x_{2}\right) \mid x_{1}^{2}=x_{2}^{3}\right\}, \mathbf{T}=\left\{\left(t, x_{1}, x_{2}\right) \mid x_{1}=\right.$ $\left.3 u^{4}+u^{2} v, x_{2}=4 u^{3}+2 u v, t=v\right\}, n_{\Gamma_{u_{0}}}\left(s_{0}\right)=\frac{\partial^{2} \Gamma}{\partial s^{2}}\left(s_{0}, u_{0}\right)$ corresponds to $\Gamma_{u_{0}}$ at $s_{0}$, $e_{\Gamma_{u_{0}}}\left(s_{0}\right)=\Gamma\left(s_{0}\right)+\frac{1}{\kappa\left(s_{0}, u_{0}\right)} \frac{\partial^{2} \Gamma}{\partial s^{2}}\left(s_{0}, u_{0}\right)$ corresponds to $\Gamma_{u_{0}}$ at $s_{0}$, and $\pi_{1}: \mathbb{R} \times \mathbb{R}^{\not z}, 0 \longrightarrow \mathbb{R}$ is the canonical projection given by $\pi_{1}\left(t, x_{1}, x_{2}\right)=t$.

We have the following result, as a corollary of Theorem 2 . 
COROLlary 3. When the parameter passes the bifurcation value two affine inflexions of order 1 (resp. affine vertices of order 1) collapse into one of order 2 and then disappear. The pair-appearance bifurcation corresponding to the opposite direction of parameter changing can also occur.

3. Proof of Theorem 2. At first we introduce some basic notions of singularity theory. For more details on the results, see $[1,3,4,6,7]$.

Let $G: \mathbb{R} \times \mathbb{R}^{\ltimes}, 0 \longrightarrow \mathbb{R}, 0$ be a function germ. We call $G$ an unfolding of $g(t)=$ $G(t, 0)$. We say that $g(t)$ has the $A_{k}$-singularity at $t$ if $g^{(p)}(t)=0$ for all $1 \leq p \leq k$, and $g^{(k+1)}(t) \neq 0$. The family $G$ is a versal unfolding of the function $g$ with the $A_{k}$-singularity if and only if the truncated Taylor expansions of $\frac{\partial G}{\partial a_{i}}(t, 0), 1 \leq i \leq n$, span the space of polynomials in $t$ of degree at most $k-1$.

We consider an unfolding $G(t, u, a)$ of the potential function $G(t, 0,0)=g(t)$. The bifurcation set is defined as

$$
\mathfrak{B}(G)=\left\{(u, a) \in U \times \mathbb{R}^{\ltimes} \mid \frac{\partial G}{\partial t}(t, u, a)=\frac{\partial^{2} G}{\partial t^{2}}(t, u, a)=0\right\} .
$$

We consider the extended unfolding $\widetilde{G}(t, u, a, c)=G(t, u, a)-c$, where $c \in \mathbb{R}$. The discriminant set is defined as

$$
\mathfrak{D}(\widetilde{G})=\left\{(u, a, c) \in U \times \mathbb{R}^{\ltimes} \times \mathbb{R} \mid G(t, u, a)=c, \frac{\partial G}{\partial t}(t, u, a)=0\right\} .
$$

The unfoldings $G$ and $\widetilde{G}$ give rise to families of bifurcation sets (resp. discriminant sets), obtained by fixing the parameter $u$. We have natural projections $\pi: U \times \mathbb{R}^{\ltimes} \longrightarrow U$ (resp. $\left.\pi_{1}: U \times \mathbb{R}^{\ltimes} \times \mathbb{R} \longrightarrow U\right)$.

TheOREM $4([3])$. Let $G(t, a, u)$ be as above. If $1, \frac{\partial G}{\partial a_{i}}(t, 0,0)(1 \leq i \leq n)$ and $\frac{\partial G}{\partial u}(t, 0,0)$ span $\mathbb{R}[t] /\left\langle t^{k}\right\rangle$ as the $\mathbb{R}$-vector space, then $\widetilde{G}$ (resp. $G$ ) is a versal unfolding of the function (resp. potential function) $g(t)$. In this case we have

(a) If $1, \frac{\partial G}{\partial a_{i}}(t, 0,0)$ span $\mathbb{R}[t] /\left\langle t^{k}\right\rangle$, the projection $\pi$ (resp. $\pi_{1}$ ) is equivalent, via a bifurcation set (resp. discriminant set) preserving diffeomorphism, to the trivial projection onto one factor of a product bifurcation (resp. discriminant) set.

(b) If $G$ is of minimal dimension $k-1$ and $1, \frac{\partial G}{\partial a_{i}}(t, 0,0)$ span $\mathbb{R}[t] /\left\langle t^{k}\right\rangle$ then the projection $\pi_{1}$ (resp. $\pi$ ) is equivalent to the projection of the standard discriminant (resp. bifurcation) set of $\widetilde{F}$ (resp. F) above onto the $a_{1}$-coordinate,

where $\widetilde{F}(t, a)= \pm t^{k+1}+a_{1} t^{k-1}+\ldots+a_{k-1} t+a_{k}$ and $F(t, a)= \pm t^{k+1}+a_{1} t^{k-1}+\ldots+a_{k-1} t$.

We now consider the Monge-Taylor map for plane curves. Throughout the remainder of this section, we fix the canonical inner product on $\mathbb{R}^{\not \models}$ which gives Euclidean structure on $\mathbb{R}^{\not \neq}$. We may consider that Euclidean structure is one of the equi-affine structures on $\mathbb{R}^{\not \models}$. For any $\gamma \in \operatorname{Imm}^{+}\left(S^{1}, \mathbb{R}^{\not \nvdash}\right)$ increasing $t$, that is the anticlockwise orientation of $S^{1}$, at each point $p$ of $\gamma\left(S^{1}\right)$ choose the positive tangent and outward normal as $x$ and $y$ axes. The curve near $p$ has a unique representation as the graph $y=f_{t}(x)$, with $f_{t}^{\prime}(x)=0$. Let $V_{p}^{k}$ be the space of polynomials in single variable of degree $d$ with $p \leq d \leq k$. We consider a map $\phi: S^{1} \longrightarrow V_{2}^{k}$ which associates to each $t \in S^{1}$ the $k$-jet of the function $f_{t}$ at 0 . We call $\phi$ a Monge-Taylor map of order $k$. 
In order to give a proof of Theorem 2, we prepare the following.

3.1. Family of affine height functions. When we study a single curve in the affine plane, we define the affine height function as follows: For a curve $\gamma: S^{1} \longrightarrow \mathbb{R}^{\not \neq}, h$ : $S^{1} \times S^{1} \longrightarrow \mathbb{R}$ is defined by $h(s, a)=\left|\gamma^{\prime}(s) a\right|$. Similarly we now define the oneparameter family of affine height functions $H: S^{1} \times U \times S^{1} \longrightarrow \mathbb{R}$ by

$$
H(s, u, a)=\left|\frac{\partial \Gamma}{\partial s}(s, u) a\right| \text {. }
$$

We also define a function $\widetilde{H}: S^{1} \times U \times S^{1} \times \mathbb{R} \longrightarrow \mathbb{R}$ by

$$
\widetilde{H}(s, u, a, v)=H(s, u, a)-v .
$$

Under this notation, we denote that

$$
\widetilde{H}_{a, v}(s, u)=H(s, u, a)-v .
$$

The discriminant set of $\widetilde{H}$ is given as

$$
\mathfrak{D}^{ \pm}(\widetilde{H})=\left\{\left(u, \lambda(s, u) \frac{\partial^{2} \Gamma}{\partial s^{2}}(s, u), \lambda(s, u)\right) \in U \times S^{1} \times \mathbb{R} \mid s \in S^{1}\right\},
$$

where $\lambda(s, u)= \pm \frac{1}{\sqrt{\frac{\partial^{2} \Gamma_{1}}{\partial s^{2}}(s, u)^{2}+\frac{\partial^{2} \Gamma_{2}}{\partial s^{2}}(s, u)^{2}}}$ and $\Gamma(s, u)=\left(\Gamma_{1}(s, u), \Gamma_{2}(s, u)\right)$.

We define a map $\Psi: U \times\left(\mathbb{R}^{\not \models}-\{\mathbf{0}\}\right) \longrightarrow U \times S^{1} \times \mathbb{R}$ by

$$
\Psi\left(u, x_{1}, x_{2}\right)=\left(u,\left(\frac{x_{1}}{\sqrt{x_{1}^{2}+x_{2}^{2}}}, \frac{x_{2}}{\sqrt{x_{1}^{2}+x_{2}^{2}}}\right), \frac{1}{\sqrt{x_{1}^{2}+x_{2}^{2}}}\right) .
$$

It is clear that $\Psi$ is a diffeomorphism and $\Psi\left(\mathcal{A} \mathcal{N}_{\Gamma}\right)=\mathfrak{D}^{+}(\widetilde{H})$, where

$$
\mathcal{A N}_{\Gamma}=\left\{\left(u, \frac{\partial^{2} \Gamma}{\partial s^{2}}(s, u)\right) \mid s \in S^{1}\right\}
$$

is the family of affine normal curves.

We consider the following singular set of $\widetilde{H}$ :

$$
\Sigma=\left\{(s, u, a, v) \in S^{1} \times U \times S^{1} \times \mathbb{R} \mid a=\lambda(s, u) \frac{\partial^{2} \Gamma}{\partial s^{2}}(s, u)\right\}
$$

and the natural projection

$$
P: \Sigma \longrightarrow U \times S^{1} \times \mathbb{R}
$$

Then the set of critical values of $P$ has the structure of the bifurcation set of $H$.

Without loss of generality, we may consider the germ at $u=0$ and $t=0$. We write $\Gamma(t, 0)=\left(t, c_{2} t^{2}+c_{3} t^{3}+c_{4} t^{4}+c_{5} t^{5}+c_{6} t^{6}+\mathbf{O}(7)\right)$ where $\mathbf{O}(k)$ denotes a smooth function on $\mathbb{R}$ vanishing at $t=0$ to order $k-1$. In particular, we assume that $c_{2} \neq 0$.

We have the following lemma by calculating derivatives of $\widetilde{H}_{a, v}$.

LEMMA 5. If $a=\left(\mp \frac{c_{3}}{\sqrt{c_{3}^{2}+4 c_{2}^{4}}}, \pm \frac{2 c_{2}^{2}}{\sqrt{c_{3}^{2}+4 c_{2}^{4}}}\right)$, the condition for $\widetilde{H}_{a}$ to have the $A_{k}$ singularity at $t=0$ is as follows:

(1) $\widetilde{H}_{a, v}$ has the $A_{1}$-singularity if and only if $5 c_{3}^{2}-4 c_{2} c_{4} \neq 0$,

(2) $\widetilde{H}_{a, v}$ has the $A_{2}$-singularity if and only if $5 c_{3}^{2}-4 c_{2} c_{4}=0$ and $7 c_{3}^{3}-4 c_{2}^{2} c_{5} \neq 0$, 
(3) $\widetilde{H}_{a, v}$ has the $A_{3}$-singularity if and only if $5 c_{3}^{2}-4 c_{2} c_{4}=0,7 c_{3}^{3}-4 c_{2}^{2} c_{5}=0$ and $21 c_{3}^{4}-8 c_{2}^{3} c_{6} \neq 0$,

(4) $\widetilde{H}_{a, v}$ has the $A_{\geq 4}$-singularity if and only if $5 c_{3}^{2}-4 c_{2} c_{4}=0,7 c_{3}^{3}-4 c_{2}^{2} c_{5}=0$ and $21 c_{3}^{4}-8 c_{2}^{3} c_{6}=0$.

We now define the function $F: S^{1} \times U \times \mathbb{R}^{\not \models} \longrightarrow \mathbb{R}$,

$$
F(s, u, x)=\frac{\partial \Gamma_{1}}{\partial s}(s, u) \sin x_{1}-\frac{\partial \Gamma_{2}}{\partial s}(s, u) \cos x_{1}-x_{2},
$$

where $x=\left(x_{1}, x_{2}\right) \in \mathbb{R}$. This is considered as a local representation of $\widetilde{H}$. We may use $F$ instead of $\widetilde{H}$.

Differentiating $F$ with respect to unfolding parameters, we obtain

$$
\begin{aligned}
& \frac{\partial F}{\partial x_{1}}(s, 0)= \pm \frac{\left(2 c_{2}\right)^{-\frac{1}{3}}}{\sqrt{c_{3}^{2}+4 c_{2}^{4}}}\left(-c_{3}+4 c_{2}^{3} t+6 c_{2}^{2} c_{3} t^{2}+\mathbf{O}(3)\right), \\
& \frac{\partial F}{\partial x_{2}}(s, 0)=-1, \\
& \frac{\partial F}{\partial u}(s, 0)= \pm \frac{\left(2 c_{2}\right)^{-\frac{1}{3}}}{\sqrt{c_{3}^{2}+4 c_{2}^{4}}}\left\{\left(2 c_{2}^{2} d_{1}+c_{3} e_{1}\right) t+\left(2 c_{2}^{2} d_{2}+c_{3} e_{2}\right) t^{2}+\mathbf{O}(3)\right\},
\end{aligned}
$$

where $\frac{\partial^{2} \Gamma_{1}}{\partial u \partial t}(t, 0)=d_{1} t+d_{2} t^{2}+\mathbf{O}(3)$ and $\frac{\partial^{2} \Gamma_{2}}{\partial u \partial t}(t, 0)=e_{1} t+e_{2} t^{2}+\mathbf{O}(3)$.

We consider the following two cases:

(1) $A_{1}$ and $A_{2}$-singularity: Since $c_{2} \neq 0,1$ and $\frac{\partial F}{\partial x_{1}}(s, 0)$ span $\mathbb{R}[t] /\left\langle t^{2}\right\rangle, F$ is always a versal unfolding. And the projection is the trivial one by Theorem 4 .

(2) $A_{3}$-singularity: If $2 c_{2}^{2} d_{2}-c_{3} e_{2} \neq 0$, the condition for a versal unfolding is that 1 , $\frac{\partial F}{\partial x_{1}}(s, 0), \frac{\partial F}{\partial x_{2}}(s, 0), \frac{\partial F}{\partial u}(s, 0)$ span $\mathbb{R}[t] /\left\langle t^{3}\right\rangle$. The condition for the projection is automatically satisfied because 1 and $\frac{\partial F}{\partial x_{1}}(s, 0)$ span $\mathbb{R}[t] /\left\langle t^{2}\right\rangle$.

For each $k \geq 2$, we consider a map $\Phi: S^{1} \longrightarrow V_{2}^{k} \times V_{0}^{k} \times V_{0}^{k}$. The first component of $\Phi$ is the Monge-Taylor map $\phi$. By a change of coordinate we may suppose that $\Gamma(t, u(0))=$ $(t, f(t))$ for some smooth $f$ with respect to the $x$ and $y$ axes. The second and third components of $\Phi$ are the Taylor expansions of $\left.\frac{\partial \Gamma_{1}}{\partial s}(t, u(0)+s)\right|_{s=0},\left.\frac{\partial \Gamma_{2}}{\partial s}(t, u(0)+s)\right|_{s=0}$ with respect to the above coordinate systems, truncated to degree $k$. Then we obtain the following theorem.

Theorem 6 ([3]). Let $X$ be a Whitney $(A)$ regular stratified subset of $V_{2}^{k} \times V_{0}^{k} \times V_{0}^{k}$. If $\Gamma(t, u)$ is a family between the generic curves $\Gamma_{0}\left(S^{1}\right)$ and $\Gamma_{1}\left(S^{1}\right)$ then it can be an arbitrarily small deformation of $\Gamma$ to a family between the same curves $\Gamma(t, 0)$ and $\Gamma(t, 1)$, and the corresponding $\Phi$ transverse to $X$.

We change the components of $\frac{\partial \Gamma}{\partial u}(t, u(0))$ to the components of $\frac{\partial^{2} \Gamma}{\partial u \partial t}(t, u(0))$ in the above map $\Phi: S^{1} \longrightarrow V_{2}^{k} \times V_{0}^{k} \times V_{0}^{k}$. By exactly the same arguments as in the proof of Theorem 3.2 in [3], we can prove the Theorem 6 .

In order to apply Theorem 6 for the proof of the assertion (1) of Theorem 2, we now seek the stratified set $X$. We take the strata to be

(1) $\left\{c_{2} x^{2}+c_{3} x^{3}+\frac{5 c_{3}^{2}}{4 c_{2}} x^{4}+\frac{7 c_{3}^{3}}{4 c_{2}^{2}} x^{5}+\frac{21 c_{3}^{4}}{8 c_{2}^{3}} x^{6}\right\} \times V_{0}^{6} \times V_{0}^{6}$ to ensure $A_{l}, l \leq 3$, 
(2) $\left\{c_{2} x^{2}+c_{3} x^{3}+\frac{5 c_{3}^{2}}{4 c_{2}} x^{4}+\frac{7 c_{3}^{3}}{4 c_{2}^{2}} x^{5}+c_{6} x^{6}\right\} \times\left\{d_{1} x+-\frac{c_{3} e_{2}}{2 c_{2}^{2}} x^{2}+d_{3} x^{3}+d_{4} x^{4}+d_{5} x^{5}+\right.$ $\left.d_{6} x^{6}\right\} \times V_{0}^{6}$ to ensure that the projection at an $A_{3}$-point is generic.

The strata are clearly of codimension at least 3 , and hence the $\Phi$ is transverse to $X$ if and only if it misses $X$, which ensures that the family $\Gamma$ is generic.

This completes the proof of assertion (1) of Theorem 2.

3.2. Family of affine distance-cubed functions. For the proof of assertion (2) of Theorem 2 , we consider the affine invariant function. When we study a single plane curve in affine plane, we define the affine distance-cubed function $d: S^{1} \times \mathbb{R}^{\not} \longrightarrow \mathbb{R}$ by $d(s, a)=\left|\frac{d \gamma}{d s}(s) \gamma(s)-a\right|$ for the curve $\gamma: S^{1} \longrightarrow \mathbb{R}^{\not}$ as the above. Similarly we now define the one-parameter family of affine distance-cubed functions $D: S^{1} \times U \times R 2 \longrightarrow \mathbb{R}$ by

$$
D(s, u, a)=\left|\frac{\partial \Gamma}{\partial s}(s, u) \Gamma(s, u)-a\right| .
$$

We also define a function, for each $a \in \mathbb{R}^{\not \nvdash}$,

$$
D_{a}(s, u)=D(s, u, a) .
$$

The bifurcation set of $D$ is

$$
\mathfrak{B}(D)=\left\{\left(u, \Gamma(s, u)+\frac{1}{\kappa(s, u)} \frac{\partial^{2} \Gamma}{\partial s^{2}}(s, u)\right) \in U \times \mathbb{R}^{\not \models} \mid \kappa(s, u) \neq 0, s \in S^{1}\right\} .
$$

$\mathfrak{B}(D)$ is the family of affine evolute. We now consider the singular set of $D$

$$
\Sigma=\left\{(s, u, a) \in S^{1} \times U \times \mathbb{R}^{\not} \mid a=\Gamma(s, u)-\lambda \frac{\partial^{2} \Gamma}{\partial s^{2}}(s, u), \lambda \in \mathbb{R}\right\}
$$

and the natural projection $P$,

$$
P: \Sigma \longrightarrow U \times \mathbb{R}^{\not \nvdash} .
$$

Then we have $\mathfrak{B}(D)=C_{P}$, where $C_{P}$ is the critical value set of $P$.

Without loss of generality we shall work at $u=0$ and $t=0$. We write $\Gamma(t, 0)=$ $\left(t, c_{2} t^{2}+c_{3} t^{3}+c_{4} t^{4}+c_{5} t^{5}+c_{6} t^{6}+c_{7} t^{7}+\mathbf{O}(8)\right)$. In particular, we assume that $c_{2} \neq 0$.

By a straightforward computation, we have the following lemma.

LEMMA 7. If $a=\left(-\frac{c_{2} c_{3}}{4 c_{2} c_{4}-5 c_{3}^{2}}, \frac{2 c_{2}^{3}}{4 c_{2} c_{4}-5 c_{3}^{2}}\right)$ the condition for $D_{a}$ to have the $A_{k}$-singularity at $t=0$ is:

(1) $D_{a}$ has the $A_{2}$-singularity if and only if $3 c_{2} c_{3} c_{4}-2 c_{3}^{3}-c_{2}^{2} c_{5} \neq 0$,

(2) $D_{a}$ has the $A_{3}$-singularity if and only if $3 c_{2} c_{3} c_{4}-2 c_{3}^{3}-c_{2}^{2} c_{5}=0$ and $2 c_{2} c_{3}^{2} c_{4}+$ $2 c_{2}^{2} c_{4}^{2}-3 c_{3}^{4}-c_{2}^{3} c_{6} \neq 0$,

(3) $D_{a}$ has the $A_{4}$-singularity if and only if $3 c_{2} c_{3} c_{4}-2 c_{3}^{3}-c_{2}^{2} c_{5}=0,2 c_{2} c_{3}^{2} c_{4}+2 c_{2}^{2} c_{4}^{2}-$ $3 c_{3}^{4}-c_{2}^{3} c_{6}=0$, and $10 c_{2} c_{3}^{3} c_{4}-10 c_{2}^{2} c_{3} c_{4}^{2}-c_{3}^{5}+c_{2}^{4} c_{7} \neq 0$,

(4) $D_{a}$ has the $A_{\geq 5}$-singularity if and only if $3 c_{2} c_{3} c_{4}-2 c_{3}^{3}-c_{2}^{2} c_{5}=0,2 c_{2} c_{3}^{2} c_{4}+$ $2 c_{2}^{2} c_{4}^{2}-3 c_{3}^{4}-c_{2}^{3} c_{6}=0$ and $10 c_{2} c_{3}^{3} c_{4}-10 c_{2}^{2} c_{3} c_{4}^{2}-c_{3}^{5}+c_{2}^{4} c_{7}=0$.

Differentiating $D$ with respect to unfolding parameters, we obtain

$$
\frac{\partial D}{\partial a_{1}}(s, 0)=\left(2 c_{2}\right)^{-\frac{1}{3}}\left(2 c_{2} t+3 c_{3} t^{2}+4 c_{4} t^{3}+\mathbf{O}(4)\right)
$$




$$
\begin{aligned}
\frac{\partial D}{\partial a_{2}}(s, 0)= & -\left(2 c_{2}\right)^{-\frac{1}{3}} \\
\frac{\partial D}{\partial u}(s, 0)= & -\left(2 c_{2}\right)^{-\frac{1}{3}}\left\{\left(e_{0}-\mu_{0} \rho\right)\right. \\
& +\left(e_{1}-n_{0}-2 c_{2} d_{0}-\mu_{1} \rho\right) t \\
& +\left(e_{2}-n_{1}-3 c_{3} d_{0}+c_{2}\left(m_{0}-2 d_{1}\right)-\mu_{2} \rho\right) t^{2} \\
& \left.+\left(e_{3}-n_{2}-4 c_{4} d_{0}+c_{2}\left(m_{1}+m_{0}-2 d_{2}\right)-3 c_{3} d_{1}-\mu_{3} \rho\right) t^{3}+\mathbf{O}(4)\right\}
\end{aligned}
$$

where $\frac{\partial \Gamma_{1}}{\partial u}(t, 0)=d_{0}+d_{1} t+d_{2} t^{2}+\mathbf{O}(3), \frac{\partial \Gamma_{2}}{\partial u}(t, 0)=e_{0}+e_{1} t+e_{2} t^{2}+e_{3} t^{3}+\mathbf{O}(4)$, $\frac{\partial^{2} \Gamma_{1}}{\partial u \partial t}(t, 0)=m_{0}+m_{1} t+m_{2} t^{2}+m_{3} t^{3}+\mathbf{O}(4), \frac{\partial^{2} \Gamma_{1}}{\partial u \partial t}(t, 0)=n_{0}+n_{1} t+n_{2} t^{2}+n_{3} t^{3}+\mathbf{O}(4)$, $\rho=\frac{1}{4 c_{2} c_{4}-5 c_{3}^{2}}$ and $\mu_{i}=c_{2}\left(2 c_{2}^{2} m_{i}-c_{3} n_{i}\right)$.

We consider the following two cases:

(1) $A_{2}$ and $A_{3}$-singularity: Since $c_{2} \neq 0,1$ and $\frac{\partial D}{\partial a_{1}}(s, 0)$ span $\mathbb{R}[t] /\left\langle t^{3}\right\rangle, D$ is always a versal unfolding if $c_{3} \neq 0$.

(2) $A_{4}$-singularity: The condition for a versal unfolding is that $1, \frac{\partial D}{\partial a_{1}}, \frac{\partial D}{\partial a_{2}}$ and $\frac{\partial D}{\partial u}$ span $\mathbb{R}[t] /\left\langle t^{4}\right\rangle$, that is $e_{3}-n_{2}-4 c_{4} d_{0}+c_{2}\left(m_{1}+m_{0}-2 d_{2}\right)-3 c_{3} d_{1}-\mu_{3} \rho \neq 0$ and $c_{3} \neq 0$. The condition for the projection is automatically satisfied by Theorem 4 .

By the above calculation, we need to consider the components of $\frac{\partial \Gamma}{\partial u}(t, u(0))$ and $\frac{\partial^{2} \Gamma}{\partial u \partial t}(t, u(0))$ in the tangent and normal directions at $\Gamma(t, u(0))$. Thus for each $k \geq 2$ we consider a map $\widetilde{\Phi}: S^{1} \longrightarrow V_{2}^{k} \times V_{0}^{k} \times V_{0}^{k} \times V_{0}^{k} \times V_{0}^{k}$. The first, second and third components of $\widetilde{\Phi}$ are the map $\Phi$. The fourth and fifth components of $\widehat{\Phi}$ are the Taylor expansions of $\left.\frac{\partial^{2} \Gamma_{1}}{\partial s \partial t}(t, u(0)+s)\right|_{s=0},\left.\frac{\partial^{2} \Gamma_{2}}{\partial s \partial t}(t, u(0)+s)\right|_{s=0}$ with respect to the above coordinate systems, truncated to degree $k$. Let $\Gamma$ be a one-parameter family between $\Gamma_{0}$ and $\Gamma_{1}$ as above. By shrinking $U$, we may suppose that $\Gamma\left(S^{1} \times U\right)$ is contained in some large ball neighbourhood of the origin $B \subset \mathbb{R}^{\not x}$. Let $P$ denote the space of polynomial maps of degree at most $d$ from $\mathbb{R}^{\not}$ to itself, and choose a sufficiently small convex neighbourhood $W$ of the zero map in $P$ so that for any $\psi_{1}, \psi_{2}, \psi_{3}, \psi_{4}$ in $W$, id $+\psi_{1}+\psi_{2}+\psi_{3}+\psi_{4}$ maps the ball $2 B$ of twice the radius of $B$ diffeomorphically onto its image (where id is the identity). Now consider $\widehat{\Gamma}: S^{1} \times U \times W^{4} \longrightarrow \mathbb{R}^{\nvdash}$ defined by $\widehat{\Gamma}\left(t, u, \psi_{1}, \psi_{2}, \psi_{3}, \psi_{4}\right)=\widetilde{\Gamma}\left(t, u, \psi_{1}, \psi_{2}\right)+\psi_{3}\left(\widetilde{\Gamma}\left(t, u, \psi_{1}, \psi_{2}\right)\right)+u \psi_{4}\left(\widetilde{\Gamma}\left(t, u, \psi_{1}, \psi_{2}\right)\right)$. Each $\left(t, u, \psi_{1}, \psi_{2} . \psi_{3}, \psi_{4}\right) \longrightarrow \widehat{\Gamma}\left(t, u, \psi_{1}, \psi_{2}, \psi_{3}, \psi_{4}\right)$ is an embedding of $S^{1}$ in $\mathbb{R}^{\not t}$. We have the associated mapping $\widehat{\Phi}: S^{1} \times U \times W^{2} \longrightarrow V_{2}^{k} \times V_{0}^{k} \times V_{0}^{k} \times V_{0}^{k} \times V_{0}^{k}$ with respect to $\widehat{\Gamma}$.

By exactly the same arguments as in the proof of Lemma 3.1 in [3], we have the following lemma.

Lemma 8. The map $\widehat{\Phi}$ is a submersion at $(t, 0,0,0,0,0)$.

By Lemma 8, we have the following theorem.

Theorem 9. Let $X$ be a Whitney (A) regular stratified subset of $V_{2}^{k} \times V_{0}^{k} \times V_{0}^{k} \times$ $V_{0}^{k} \times V_{0}^{k}$. If $\Gamma(t, u)$ is a family between the generic curves $\Gamma_{0}\left(S^{1}\right)$ and $\Gamma_{1}\left(S^{1}\right)$ then it can be an arbitrarily small deformation of $\Gamma$ to a family between the same curves $\Gamma(t, 0)$ and $\Gamma(t, 1)$, and the corresponding $\widetilde{\Phi}$ transverse to $X$. 
Since the proof of Theorem 9 is analogous to that of Theorem 6 (Theorem 3.2 in [3]), we omit it.

For applying Theorem 9, we now adopt the canonical Whitney stratification of the following algebraic set:

(1) $\left\{c_{2} x^{2}+c_{4} x^{4}-\frac{2 c_{4}^{2}}{c_{2}} x^{6}\right\} \times V_{0}^{7} \times V_{0}^{7} \times V_{0}^{7} \times V_{0}^{7}$ to ensure $A_{l}, l \leq 3$,

(2) $\left\{c_{2} x^{2}+c_{4} x^{4}-\frac{2 c_{4}^{2}}{c_{2}} x^{6}+c_{7} x^{7}\right\} \times V_{0}^{7} \times\left\{e_{0}+e_{1} x+e_{2} x^{2}+\tilde{e}_{3} x^{3}+e_{4} x^{4}+e_{5} x^{5}+e_{6} x^{6}+\right.$ $\left.e_{7} x^{7}\right\} \times V_{0}^{7} \times V_{0}^{7}$, where $\tilde{e}_{3}=\left(n_{2}+4 c_{4} d_{0}-c_{2}\left(m_{1}+m_{0}-2 d_{2}\right)+\frac{2 c_{2}^{2} m_{3}}{2 c_{4}}\right)$ to ensure that the projection at an $A_{3}$-point is generic.

The strata are clearly of codimension at least 3 , and hence the map $\Phi$ is transverse to $X$ if and only if it misses $X$, which ensures that the family $\Gamma$ is generic.

This completes the proof of assertion (2) of Theorem 2.

4. Examples. The situation described in Theorem 2 is depicted as follows.

The first example is the bifurcation of affine inflexions. So we draw some affine normal curves. The original family of curves is

$$
\gamma(t)=(\cos 3 t+a \cos t-10 \sin t+\cos 2 t, \sin 3 t+a \sin t+10 \cos t-\sin 2 t),
$$

where $a$ is the parameter. The real lines of Figure 1 are the affine normal curves of $\gamma$. We draw affine normal curves for the parameters $-10,-11.0,-12.0,-12.5,-12.7$ and -12.9 . When we draw both of the curve $\gamma$ and the affine normal curve of $\gamma$, the affine normal curve of $\gamma$ is drawn as very small pictures. So we draw only the affine normal curves for each parameter $a$. Since the ordinary affine inflexion corresponds to the ordinary cusp of the affine normal curve from Theorem 1, we can recognize the number of affine inflexions by counting the number of cusps of the affine normal curve.
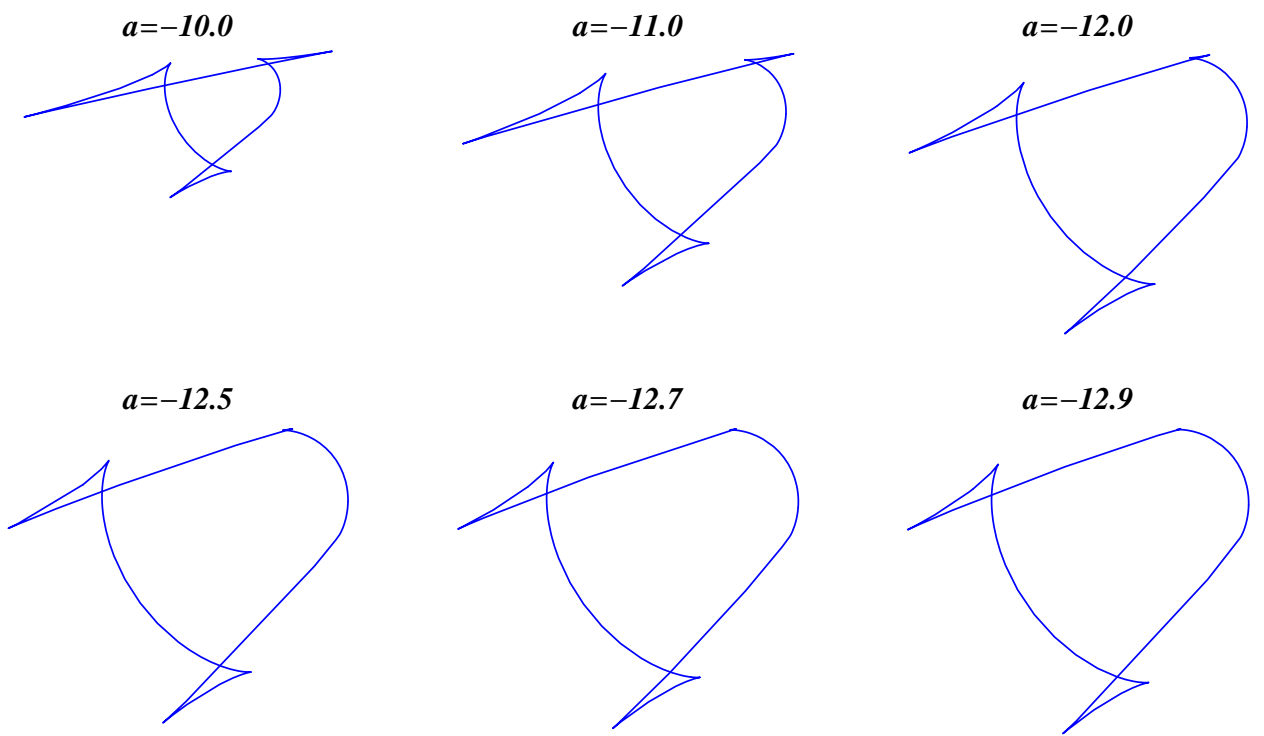

Figure 1 
The second example is the bifurcation of affine vertices. The original family of curves is

$$
\gamma(t)=(\cos 2 t-\cos (t+a), \sin 2 t+\sin t)
$$

They are drawn by the dotted curves. We draw affine evolutes of $\gamma$ for the parameters $1.9,2.0,2.1,2.2,2.3$ and 2.4. Affine evolutes are drawn by the real curves.
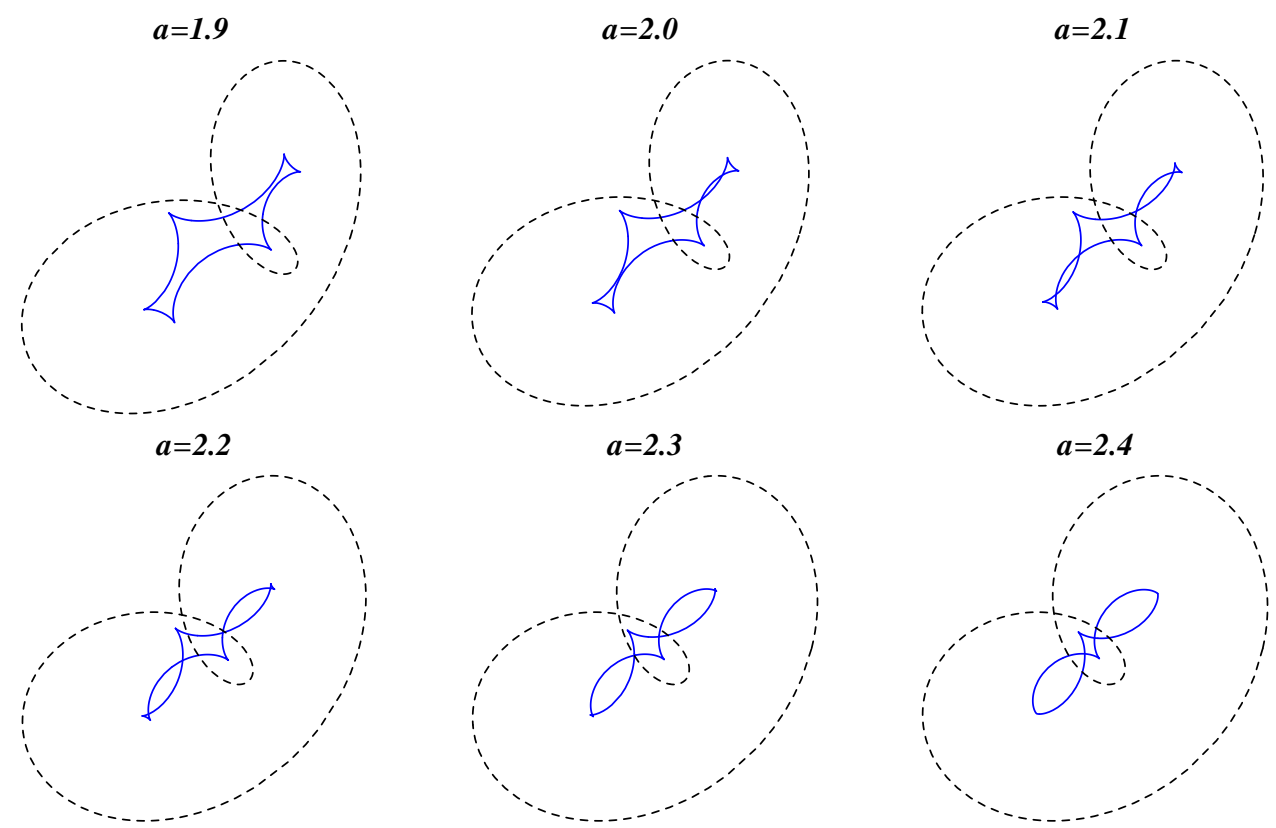

Figure 2

Acknowledgments. The author would like to thank Professor S. Izumiya for proposing the problems in this paper and for helpful advices.

\section{References}

[1] V. I. Arnol'd, Wave front evolution and equivariant Morse lemma, Comm. Pure Appl. Math. 29 (1976), 557-582.

[2] W. Blaschke, Vorlesungen über Differentialgeometrie II, Springer, Berlin, 1923.

[3] J. W. Bruce, Isotopies of generic plane curves, Glasgow Math. J. 24 (1983), 195-206.

[4] J. W. Bruce and P. J. Giblin, Curves and Singularities. A Geometrical Introduction to Singularity Theory, Cambridge Univ. Press, Cambridge, 1984.

[5] D. L. Fidal, The existence of sextactic points, Math. Proc. Cambridge Philos. Soc. 96 (1984), 433-436.

[6] D. L. Fidal and P. J. Giblin, Generic 1-parameter families of caustics by reflexion in the plane, Math. Proc. Cambridge Philos. Soc. 96 (1984), 425-432.

[7] C. G. Gibson, Singular Points of Smooth Mappings, Pitman Research Notes in Mathematics 25, Pitman Publ., London, 1979.

[8] S. Izumiya and T. Sano, Generic affine differential geometry of plane curves, Proc. Edinburgh Math. Soc. (2) 41 (1998), 315-324.

[9] K. Nomizu and T. Sasaki, Affine Differential Geometry. Geometry of Affine Immersions, Cambridge Tracts in Math. 111, Cambridge Univ. Press, Cambridge, 1994. 